\title{
Rhabdomyolysis-induced acute kidney injury was ameliorated in NLRP3 KO mice via alleviation of mitochondrial lipid peroxidation in renal tubular cells
}

\author{
Yang Gyun Kim, Ju-Young Moon, Hyeon Seock Hwang, Jin Sug Kim, Kyung Hwan Jeong, Sang-ho Lee
}

Division of Nephrology, Department of Internal Medicine, Kyung Hee University School of Medicine, Seoul, Korea

Background: A recent study showed early renal tubular injury in rhabdomyolysis-induced acute kidney injury (RIAKI) was ameliorated in NOD-like receptor pyrin domain-containing protein 3 (NLRP3) KO mice. However, the precise mechanism has not been determined. Therefore, we investigated the role of NLRP3 in renal tubular cell in RIAKI.

Methods: A glycerol-induced RIAKI was generated in NLRP3 KO and wild type mice. The mice were euthanized 24 hours after glycerol injection, and both kidneys and plasma were collected. HKC-8 cells were treated with ferrous myoglobin to mimic the rhabdomyolysis environment.

Results: The glycerol injection led to increase serum creatinine, aspartate aminotransferase (AST), renal kidney injury molecule-1 (KIM-1), renal tubular necrosis, and apoptosis. Renal injuries were attenuated in NLRP3 KO mice, while muscle damage and renal neutrophil recruitment did not differ from wild type mice. Following glycerin injection, cleaved caspase-3, poly(ADP-ribose) polymerase (PARP), and acyl-CoA synthetase long chain family member 4 (ACSL4) were increased, and glutathione peroxidase 4 (GPX-4) was decreased in RIAKI kidney, and these changes were alleviated in NLRP3 KO kidney. NLRP3 was up-regulated, and cell viability was suppressed in HKC-8 cells under ferrous myoglobin circumference. Myoglobin induced apoptosis and lipid peroxidation was significantly lessened in siNLRP3 treated HKC-8 cells. Myoglobin caused to reduce mitochondrial membrane potential, increase mitochondrial fission, reactive oxygen species, and lipid peroxidation, which were recovered in NLRP3 depleted HKC-8 cells.

Conclusions: NLRP3 depletion ameliorated renal tubular injuries in the murine glycerol-induced AKI model. NLRP3 absence affected to improve tubular cell viability via attenuation of myoglobin-induced mitochondrial injuries and lipid peroxidation, which might be the critical factor in protecting the kidney. 